\title{
Instrumento para determinar el cambio en los hábitos de consumo durante la pandemia causada por el COVID-19
}

\author{
Fecha de recepción: 05-01-2021 • Fecha de aceptación: 03-02-2021 • Fecha de publicación: 10-02-2021
}

Andrés Francisco López Gómez ${ }^{1}$ Instituto Tecnológico Superior Universitario España, Ecuador andresf.lopezg@iste.edu.ec https://orcid.org/0000-0003-4068-9837

María del Carmen Gómez Romo² Universidad Técnica de Ambato, Ecuador mariadgomez@uta.edu.ec https://orcid.org/0000-0003-1641-5465

\section{RESUMEN:}

Los cambios recurrentes existentes, como la pandemia del 2020, obligan a analizar modificaciones en el comportamiento, mediante los cuales, los seres humanos han realizado adaptaciones en su conducta y sobre múltiples desempeños. El presente estudio se enfoca en el desarrollo de un instrumento para el estudio de la variación del consumo de bienes, servicios y la predisposición al comportamiento altruista. Se desarrolló un cuestionario de 22 preguntas diseñado para caracterizar al sujeto de estudio y recopilar información acerca de las variables de control. El instrumento fue validado mediante el coeficiente de Alpha de Cronbach. Las variables de control permitieron caracterizar al sujeto de estudio, al tiempo que las preguntas de la encuesta se definieron de tal manera que permitieron contar con una visión global de los cambios que se han suscitado en el comportamiento del consumidor desde que inició la pandemia. Se identificó que un $24.6 \%$ de familias mantuvo sus ingresos y un $4.3 \%$ de familias los incrementaron durante el confinamiento. Entre los resultados se puede apreciar que los hábitos de consumo cambiaron y qué comercio, mediante aplicaciones móviles, aumentó, pero sin representar una disrupción. El instrumento respondió positivamente a los procesos de validación y queda a disposición de investigadores. 
PALABRAS CLAVE: comportamiento del consumidor, COVID-19, preferencias de consumo, instrumento de investigación.

\begin{abstract}
The existing recurrent changes, such as the 2020 pandemic, make it necessary to analyze modifications in behavior, through which human beings have made adaptations in their behavior and on multiple performances. The present study focuses on the development of an instrument for the study of the variation in the consumption of goods and services and the predisposition to altruistic behavior. A 22-question questionnaire designed to characterize the study subject and collect information about control variables was developed. The instrument was validated using Cronbach's Alpha coefficient. The control variables allowed characterization of the study subject, while the survey questions were defined in such a way as to provide an overview of the changes that have occurred in consumer behavior since the pandemic began. It was identified that $24.6 \%$ of families maintained their income and $4.3 \%$ of families increased their income during the confinement. Among the results, it can be seen that consumer habits changed and that commerce, through mobile applications, increased, but without representing a disruption. The instrument responded positively to the validation process and is now available to researchers.
\end{abstract}

\title{
KEYWORDS: consumer behavior, COVID-19, consumer preferences, religious behavior, research
} instrument. 


\section{Introducción}

Los cambios constantes a los que ha estado sujeto el mundo ha exigido al ser humano mantenerse en un estado de alerta para conquistar nuevos conocimientos y realizar cada vez nuevas adaptaciones dentro de sus múltiples desempeños. Este patrón de cambio recurrente trae consigo mayores exigencias al enfrentar nuevas realidades, como es el caso de la pandemia causada por el COVID-19, iniciada en el mes de marzo 2020. En este escenario, actividades de todo tipo, sean productivas, de servicios o comerciales se han visto seriamente afectadas, de tal manera que, se convierte en una cuestión imperativa encontrar nuevas maneras de hacer las cosas. La afección se ha extendido a todo tipo de organizaciones, sin importar su tamaño, y por supuesto a todo tipo de personas, sin importar la ubicación geográfica.

La presente investigación desarrolla un instrumento que contribuye a abordar cuestiones como ¿qué efecto ha tenido la cuarentena en la preferencia de visita de grandes superficies comerciales y pequeños comercios? ¿qué ocurrió con la concurrencia religiosa? ¿qué ocurrió con el comportamiento altruista? ¿cuál es el nivel de educación del sujeto de estudio y los cambios de comportamiento observados? ¿cómo ha cambiado la predisposición al ocio familiar? en base a las interrogantes enunciadas se plantea que el principal objetivo de la encuesta es proporcionar información que permita determinar los cambios conductuales originados a partir de la gran cuarentena de 2020.

De manera general, se presupone que como la economía se ha contraído, los comportamientos de consumo se habrán ajustado a las limitaciones. Factores como el miedo al contagio, al desempleo, o los ajustes presupuestarios potencialmente derivarán en cambios de conductas de consumo. Se considera de especial interés determinar los cambios en las preferencias de los lugares de adquisición de los productos de primera necesidad, el comportamiento religioso gregario, y la conducta altruista manifestada en donaciones.

La crisis actual afecta tanto a la oferta como a la demanda, así pues, el inminente recrudecimiento de la pandemia a nivel global ha provocado que los países tomen cada vez medidas más extremas en contra de la recesión económica (Clavellina, 2020; Gourinchas, 2020). Para que las medidas adoptadas surtan los efectos deseados estas deberán ser coordinadas mediante un liderazgo fuerte a nivel sanitario, empresarial, de gobierno y ciudadano (Nicola, 2020). La pandemia ha generado múltiples fenómenos de mercado para los que no estaba preparada la población porque corresponden a eventos raros del ámbito de la epidemiología (Maldonado, 2016). Los eventos raros, por naturaleza son difíciles, por no asumir que imposibles de predecir. Por tal motivo urge recopilar datos que permitan contemplar con perspectivas oportunas, quiénes se han visto afectados y de qué modo.

En el presente estudio se ha diseñado una encuesta que indaga los distintos cambios de conducta generados en consumidores domésticos, tomando en cuenta el nivel de estudios, género, situación laboral y su participación en actividades religiosas. Las condiciones de la cuarentena podrían haber sido ideales para estudiar fenómenos como el pesimismo defensivo y el optimismo, mas según Fernández (2001), ya se han dedicado muchos trabajos a comprobar la funcionalidad y 
efectividad de sus estrategias. Sin embargo, a pesar de que incrementar la comprensión acerca de la influencia de los rasgos de personalidad en las tendencias de consumo sería útil, poco puede hacerse para modificar los rasgos de personalidad, peor aún carácter o temperamento (Martínez 2002), al menos en poblaciones enteras es inviable.

Por el contrario, es completamente factible afectar la distribución del nivel de educación en una población por medio de la implementación de políticas públicas. Si bien, por respeto al derecho a la libertad de cultos no se pueden interferir en prácticas religiosas, comprender cómo actúa la gente que profesa activamente una fe puede servir para optimizar la colaboración pública con instituciones de carácter religioso, en pro del beneficio de la ciudadanía en general. La segmentación, caracterización y análisis comportamental de los individuos en la casuística pandémica de 2020, es de trascendental importancia, en previsión de la multiplicación y aceleración de la ocurrencia de fenómenos globales, inherentes a la sobrepoblación planetaria.

\section{Metodología}

El abordaje del diseño del instrumento procuró una estructura que cuida la maximización de la utilidad en la recopilación de información, y que contribuya a la toma de decisiones para orientarlas a la recuperación económica post pandemia. Es importante recopilar datos que, luego puedan convertirse en información, así pues, vale mencionar por ejemplo que el pronóstico del incremento del desempleo en Estados Unidos, en el sector de comercio al por menor equivalente a una reducción del $5.4 \%$ de plazas de empleo, solo es superado por la reducción estimada de 5.6\% plazas de empleo en el sector de ocio y turismo (Hatzius, Philips, Mericle \& Struyven, 2020). Las divergencias entre las dinámicas de los mercados maduros norteamericanos en comparación con los mercados en vías de desarrollo Latinoamericanos, instan poner atención al problema sin asumir que el fenómeno se arrollará de igual manera.

Para tomar en cuenta el comportamiento religioso, en el presente instrumento se consideró la premisa de que existe una correlación entre la ética de las religiones y las decisiones económicas de quienes las profesan (Weber, 2012). Evitar que una economía en crisis colapse requiere de un consumo responsable. El nivel de educación es relevante para insertar ese tipo de conceptos abstractos como lo es la responsabilidad social en las compras, asunto que está ligado con el nivel de educación del individuo (Bigné, 2006). En función de lo que se descubra con la aplicación de este instrumento se contemplará los ajustes pertinentes para mejorar el rendimiento del instrumento tanto con fines académicos como de gestión pública.

Se utilizó una metodología cuali-cuantitativa porque recoge información a partir de preguntas formuladas tomando en cuenta criterios de expertos, y que permitirá ofrecer información cuantificada a través de gráficas estadísticas que describan el fenómeno investigado (Rodríguez Rivadeneira, 2015). Se diseñó un instrumento que será aplicado mediante una encuesta que responde a las preguntas: ¿en qué tipo de lugar prefieren comprar productos de consumo doméstico las personas residentes en la ciudad de Ambato? y ¿cómo modificó la cuarentena las preferencias en relación los lugares de compra? El propósito de la encuesta es evidenciar los cambios en los comportamientos de consumo antes de la cuarentena, y a partir de esta. 
Tomando en cuenta la cantidad de unidades de análisis, las variables y la temporalidad, se propone la aplicación del instrumento diseñado dentro de una investigación cuasi experimental de carácter multivariado en razón de que intervendrán una cantidad considerable de variables que forman parte de este estudio transversal. Con el propósito indicado, este instrumento se estructuró con 22 preguntas, organizado en 6 preguntas para la caracterización del sujeto de estudio, y 17 preguntas para el estudio de los aspectos relacionados con los cambios de comportamiento en el consumo de determinados bienes y servicios. Las variables de caracterización son: edad, género, educación, condición de trabajo y tipo de trabajo. Los indicadores de cambio de comportamiento de consumo giran alrededor del comportamiento gregario religioso, altruismo, acondicionamiento físico y ocio familiar, preferencias de lugar de compra de tres categorías de tipos de productos de consumo doméstico, conforme a la costumbre de los pobladores de la ciudad en estudio: frutas y verduras, pan y leche, insumos de abasto (arroz, azúcar, granos, enlatados, productos de limpieza, etc.).

Con el fin de verificar la validez del instrumento se aplicó en un ensayo piloto de 60 personas, y de acuerdo a esos resultados se hizo reajustes. Finalmente, para verificar su efectividad, se aplicó en 933 personas que se consideró representativa de los habitantes de la ciudad de Ambato que incluyó a 6 barrios con diferente ubicación y características. Las condiciones de distanciamiento social vigentes derivaron en que la encuesta no pueda correrse de forma presencial. El método de aplicación de la encuesta fue digital, mediante Google Forms, por lo tanto, la investigación se destinó a personas con acceso a dispositivos tecnológicos. El número de encuestas efectivas fue de 392 con lo que se termina que la tasa de respuesta fue del $42 \%$ como se puede observar en la Tabla 1.

Tabla 1.

Distribución de la aplicación de la encuesta en 6 barrios de la ciudad de Ambato

\begin{tabular}{|l|l|l|}
\hline Barrios & Encuestados & $\begin{array}{l}\text { Encuestas } \\
\text { efectivas }\end{array}$ \\
\hline A & 148 & 67 \\
\hline B & 167 & 59 \\
\hline C & 193 & 63 \\
\hline D & 132 & 63 \\
\hline E & 124 & 64 \\
\hline F & 169 & 77 \\
\hline Total & 933 & 392 \\
\hline
\end{tabular}

Fuente: elaboración propia

La recolección de las respuestas entorno a las preguntas del comportamiento de consumo antes de la pandemia y a partir de la pandemia, se estructuraron con escala de Likert de cinco niveles. En cuanto a la recolección de las respuestas producto de las preguntas de las variables de preferencias de lugar de consumo, se contemplaron cinco categorías que condensan la variedad de opciones que disponen los consumidores de la ciudad de Ambato: plazas o mercados municipales, tiendas de barrio, supermercados, llamadas o mensajes telefónicos, aplicaciones 
móviles.

En la Tabla 2 se detalla el contenido de la encuesta de manera específica. Ahí se aprecia que tiene 1 pregunta para edad, 1 para género, 1 para nivel de educación, 2 para situación laboral, y 1 para variación de ingresos. Estas seis preguntas sirven para caracterizar la población objeto de estudio. Luego se tienen 17 preguntas que se definieron como estilo de vida entre las que están la asistencia a reuniones religiosas, predisposición para hacer donaciones (altruismo), acondicionamiento físico, ocio familiar, preferencia de lugares de compra para frutas y verduras, pan y leche, y productos de abasto casero. Estas preguntas abarcan los escenarios antes y después de la pandemia. De esta manera se configuran las 22 preguntas del instrumento.

Tabla 2.

Estructura de la encuesta

\begin{tabular}{|c|c|c|c|}
\hline $\begin{array}{l}\text { Valiables } \\
\text { primarias }\end{array}$ & $\begin{array}{l}\text { Variables } \\
\text { secundarias }\end{array}$ & Preguntas & Opciones \\
\hline \multirow{6}{*}{$\begin{array}{l}\text { Caracterización } \\
\text { de la población }\end{array}$} & Edad & Edad en años & Sin opciones \\
\hline & Género & Genero & $\begin{array}{l}\text { 1. Femenino } \\
\text { 2. Masculino }\end{array}$ \\
\hline & Nivel de educación & Nivel de educación & $\begin{array}{l}\text { 1. Básica } \\
\text { 2. Bachillerato } \\
\text { 3. Superior } \\
\text { 4. Posgrado }\end{array}$ \\
\hline & \multirow[t]{2}{*}{ Situación laboral } & ¿Está actualmente trabajando? & $\begin{array}{l}\text { 1. Si } \\
\text { 2. No } \\
\end{array}$ \\
\hline & & Está trabajando como & $\begin{array}{l}\text { 1. Empleado público } \\
\text { 2. Empleado privado } \\
\text { 3. Negocio propio } \\
\text { 4. Nuevo emprendimiento } \\
\text { en este año }\end{array}$ \\
\hline & $\begin{array}{l}\text { Variación en los } \\
\text { ingresos por } \\
\text { pandemia } \\
\end{array}$ & Sus ingresos a partir de la pandemia & $\begin{array}{l}\text { 1. Se mantuvieron } \\
\text { 2. Aumentaron } \\
\text { 3. Disminuyeron }\end{array}$ \\
\hline
\end{tabular}




\begin{tabular}{|c|c|c|c|}
\hline \multirow[t]{10}{*}{ Estilo de Vida } & \multirow[t]{2}{*}{$\begin{array}{l}\text { Comportamiento } \\
\text { gregario religioso }\end{array}$} & $\begin{array}{l}\text { Antes de la pandemia ¿asistía a algún tipo de } \\
\text { reuniones de religiosas o espirituales? }\end{array}$ & \multirow{10}{*}{$\begin{array}{l}\text { Escala de Likert de } 5 \\
\text { niveles: Nunca - Rara vez } \\
\text { - A veces - Casi siempre - } \\
\text { Siempre }\end{array}$} \\
\hline & & $\begin{array}{l}\text { A partir de la pandemia utilizando algún medio } \\
\text { tecnológico, ¿asiste a algún tipo de reuniones } \\
\text { religiosas o espirituales? }\end{array}$ & \\
\hline & \multirow[t]{2}{*}{ Altruismo } & $\begin{array}{l}\text { Antes de la pandemia, ¿hacía donaciones a alguna } \\
\text { causa? }\end{array}$ & \\
\hline & & $\begin{array}{l}\text { A partir de la pandemia, ¿hace donaciones a alguna } \\
\text { causa? }\end{array}$ & \\
\hline & \multirow[t]{2}{*}{$\begin{array}{l}\text { Acondicionamiento } \\
\text { físico }\end{array}$} & $\begin{array}{l}\text { Antes de la pandemia, ¿asistía a gimnasios o } \\
\text { tomaba clases de entrenamiento físico? }\end{array}$ & \\
\hline & & $\begin{array}{l}\text { A partir de la pandemia, utilizando cualquier medio } \\
\text { tecnológico, ¿toma clases de entrenamiento } \\
\text { personal? }\end{array}$ & \\
\hline & \multirow[t]{4}{*}{ Ocio familiar } & $\begin{array}{l}\text { Antes de la pandemia, ¿dedicaba tiempo de ocio } \\
\text { para recreación en familia? }\end{array}$ & \\
\hline & & $\begin{array}{l}\text { A partir de la pandemia, ¿dedicó tiempo de ocio para } \\
\text { recreación en familia? }\end{array}$ & \\
\hline & & $\begin{array}{l}\text { Antes de la pandemia, ¿invertía dinero para la } \\
\text { recreación en familia? }\end{array}$ & \\
\hline & & $\begin{array}{l}\text { A partir de la pandemia, ¿invierte dinero para la } \\
\text { recreación en familia? }\end{array}$ & \\
\hline \multirow{6}{*}{$\begin{array}{l}\text { Preferencia } \\
\text { de lugares de } \\
\text { compra por } \\
\text { producto }\end{array}$} & \multirow[t]{2}{*}{ Frutas y verduras } & $\begin{array}{l}\text { Antes de la pandemia, para comprar frutas y } \\
\text { verduras, acudía a: }\end{array}$ & \multirow{6}{*}{$\begin{array}{l}\text { 1. Plazas o mercados } \\
\text { municipales } \\
\text { 2. Supermercados } \\
\text { 3. Llamadas o mensajes } \\
\text { telefónicos } \\
\text { 4. Aplicaciones móviles }\end{array}$} \\
\hline & & $\begin{array}{l}\text { A partir de la pandemia, para comprar futas y } \\
\text { verduras, acude a: }\end{array}$ & \\
\hline & \multirow[t]{2}{*}{ Pan y leche } & $\begin{array}{l}\text { Antes de la pandemia, para comprar pan y leche, } \\
\text { acudía a: }\end{array}$ & \\
\hline & & $\begin{array}{l}\text { A partir de la pandemia, para comprar pan y leche, } \\
\text { acude a: }\end{array}$ & \\
\hline & \multirow{2}{*}{$\begin{array}{l}\text { Arroz, azúcar, } \\
\text { granos, enlatados, } \\
\text { productos de } \\
\text { limpieza, etc. }\end{array}$} & $\begin{array}{l}\text { Antes de la pandemia, para comprar abastos (arroz, } \\
\text { azúcar, granos, enlatados, productos de limpieza, } \\
\text { etc.), acudía a: }\end{array}$ & \\
\hline & & $\begin{array}{l}\text { A partir de la pandemia, para comprar abastos } \\
\text { (arroz, azúcar, granos, enlatados, productos de } \\
\text { limpieza, etc.), acude a: }\end{array}$ & \\
\hline
\end{tabular}

Fuente: elaboración propia

\section{Resultados}

El instrumento de investigación (encuesta) fue validado estadísticamente con el apoyo de software SPSS (versión 24) con el que se obtuvo un coeficiente Alfa de Cronbach de 0,811 que indica una alta fiabilidad del instrumento (Levin, 2004; Lind, 2005). La aplicación definitiva de la encuesta se llevó a cabo después de una prueba piloto aplicada a 60 personas en el mes de agosto, después 
de la cual, mejoró la comprensión de las preguntas. La encuesta definitiva fue aplicada en el mes de septiembre de 2020. Los datos recolectados justifican la presencia de insumos informativos que dan lugar a la representación e interpretación de los resultados a través de herramientas de estadística descriptiva.

Los datos obtenidos permitieron establecer que el $51 \%$ de la población a la que se aplicó la encuesta es de género femenino, mientras que el $48.6 \%$ del género masculino. Esta participación porcentual constituye un indicio favorable de representatividad y equidad, puesto que conforme al censo del INEC (2010), la población del cantón de Ambato está constituida por un 51.55 de mujeres y un 48.45 de hombres.

En cuanto al nivel educativo la muestra, se evidencia un sesgo hacia clases educadas, puesto que entre los encuestados el $63.4 \%$ cuenta con educación superior, el $25.4 \%$ cuenta estudios de posgrado, el 10,6\% cuenta con título de bachiller y el $0.6 \%$ con educación básica. Por otro lado, el $29.6 \%$ de los encuestados actualmente no labora, mientras que el $70.4 \%$ manifiesta que actualmente se encuentran laborando. De ellos el $30.4 \%$ son empleados privados, el $30.4 \%$ cuentan con un negocio propio, el $20.8 \%$ son empleados públicos y el $18.4 \%$ trabajan en un nuevo emprendimiento que inició en el último año.

A su vez, el $71 \%$ de los encuestados aseguran que a partir de la pandemia sus ingresos disminuyeron. Para el $24 \%$ sus ingresos se mantuvieron mientras que para el $4.3 \%$ de los encuestados la pandemia les significó un incremento en sus ingresos.

Los resultados de los indicadores referentes al cambio de comportamiento de consumo se resumieron en cinco gráficos de barras que facilitan su apreciación de modo comparativo antes y después de la cuarentena.

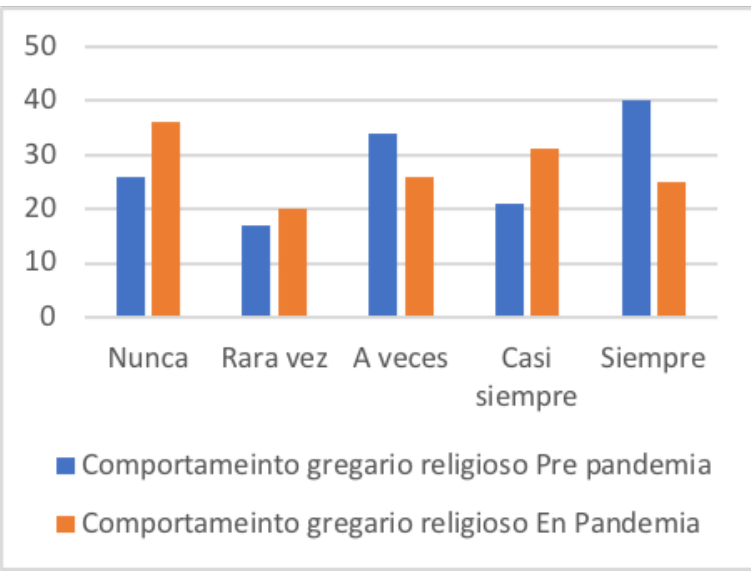

Figura 1. Comportamiento gregario religioso

Fuente: elaboración propia

De acuerdo con la Figura 1, el comportamiento gregario religioso se ha visto drásticamente modificado, con una importante disminución en tiempos de cuarentena. De igual manera las donaciones han disminuido (Figura 2). 


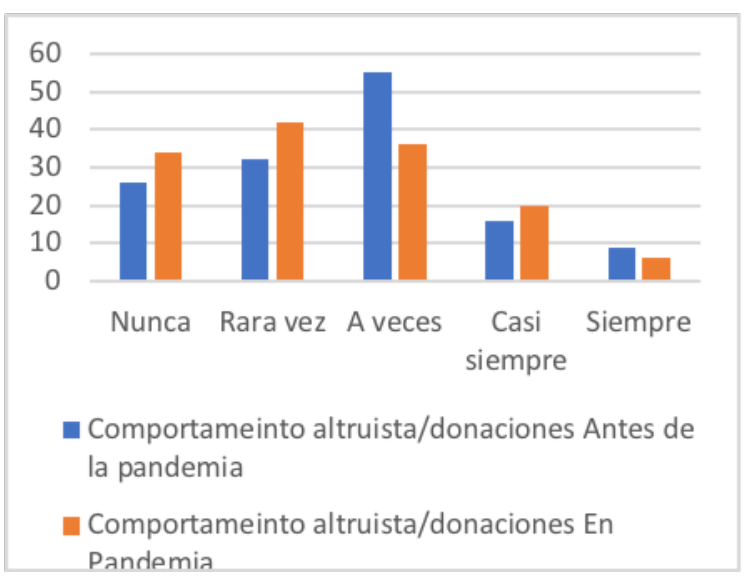

Figura 2. Comportamiento altruista/donaciones

Fuente: elaboración propia

Aunque el tiempo dedicado al ocio familiar no bajó notoriamente (Figura 3), el rubro destinado para ocio si disminuyó significatimente, tal como se evidencia en la Figura 4.

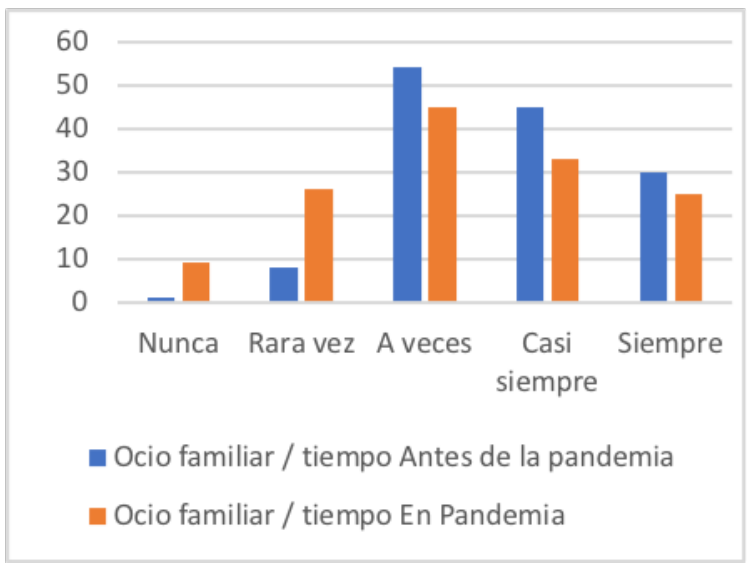

Figura 3. Tiempo de ocio familiar

Fuente: elaboración propia 


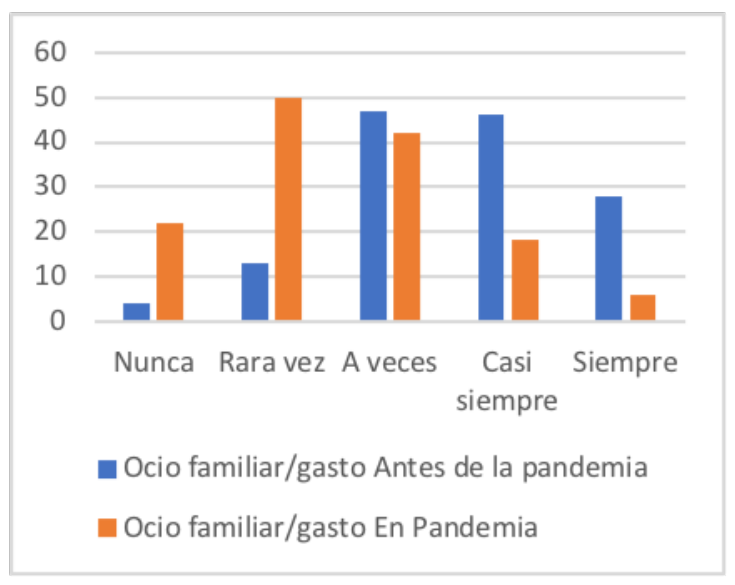

Figura 4. Gasto en ocio familiar

Fuente: elaboración propia

El impacto en el consumo de servicios de entrenamiento personal (Figura 5) se ha visto menos castigado que el dedicado al ocio familiar.

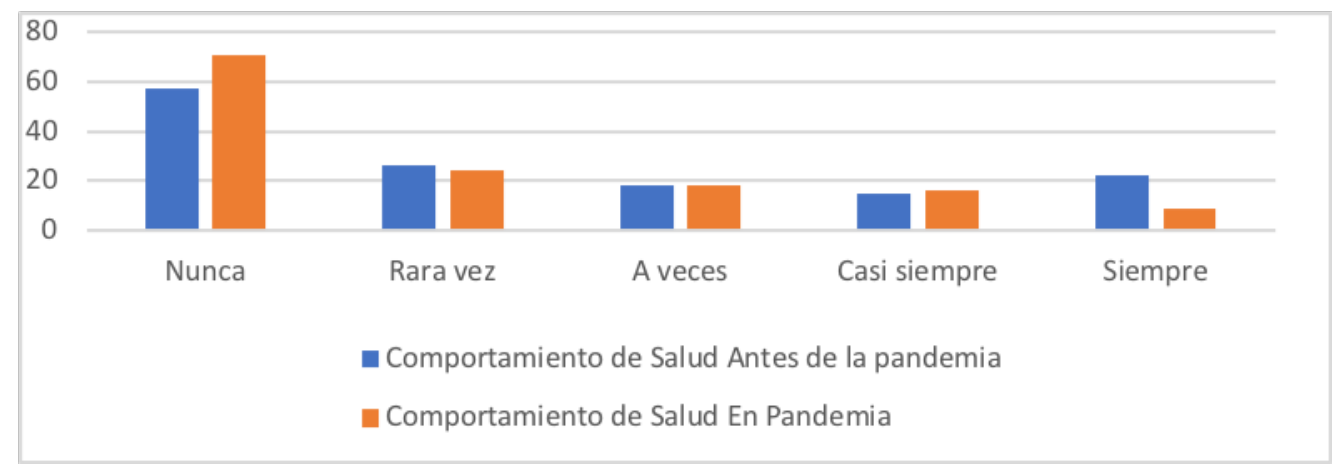

Figura 5. Consumo de entrenamiento fisico

Fuente: elaboración propia

De manera general puede apreciarse ajuste en todos los mercados. Las Figuras 6, 7 y 8 representan la cantidad de incidencias de cambio de preferencias de los lugares de compra en la muestra, como se indica a continuación. 


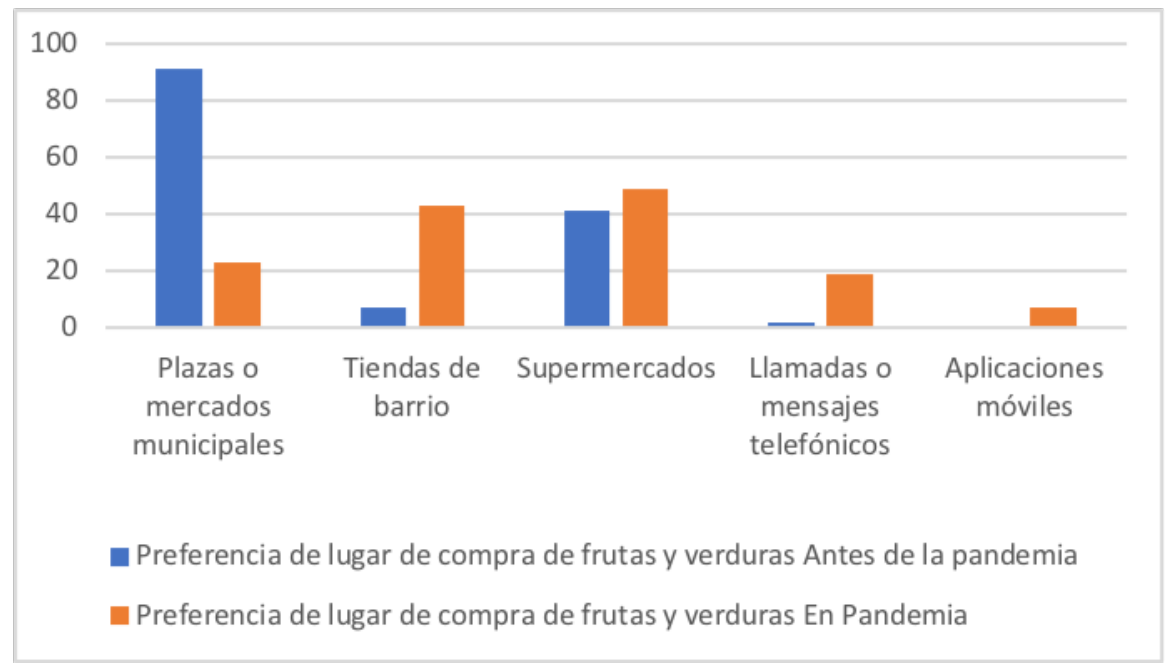

Figura 6. Preferencia de lugar de compra de frutas y verduras

Fuente: elaboración propia

El sector más golpeado es el del comercio popular campesino que se desarrolla en las plazas y mercados municipales (Figura 6 y Figura 7).

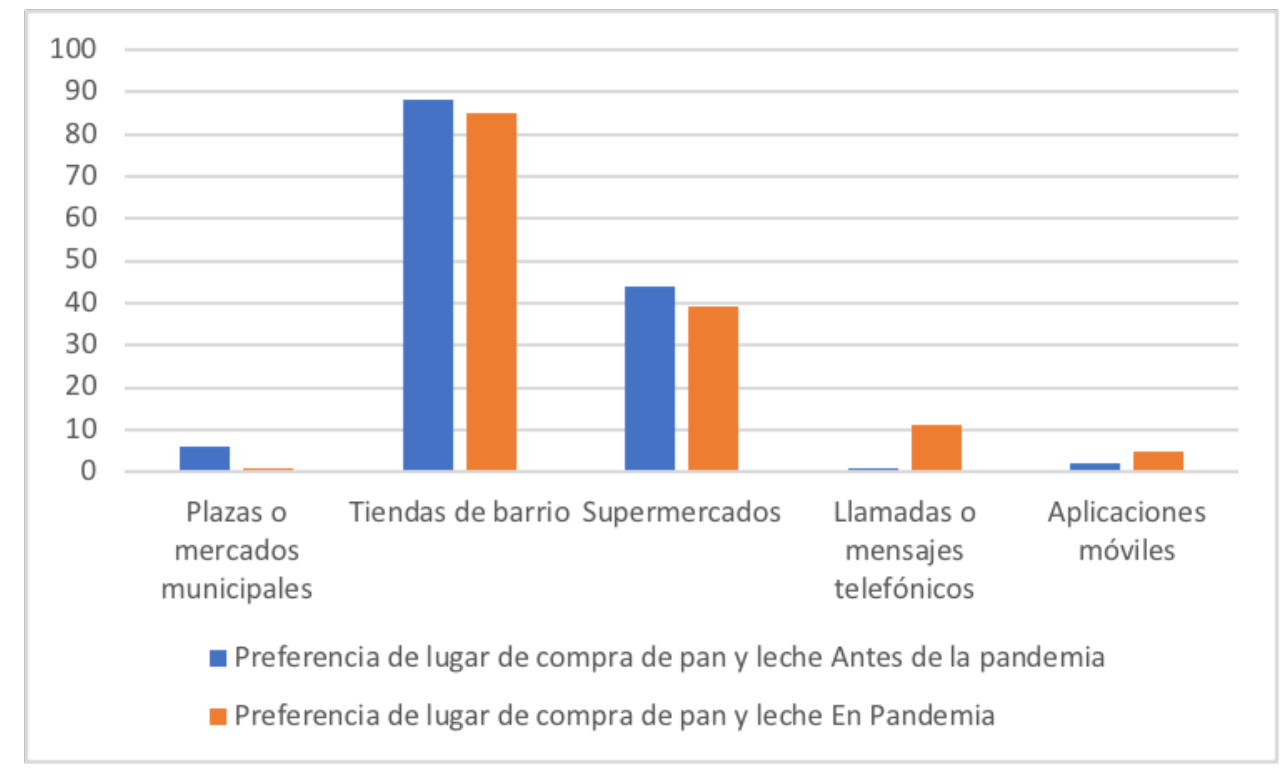

Figura 7. Preferencia de lugar de compra de pan y leche

Fuente: elaboración propia

Los servicios por medio de llamadas o mensajes telefónicos y las aplicaciones móviles han provocado un ajuste quitándole un margen de participación a tiendas de barrio y supermercados. 


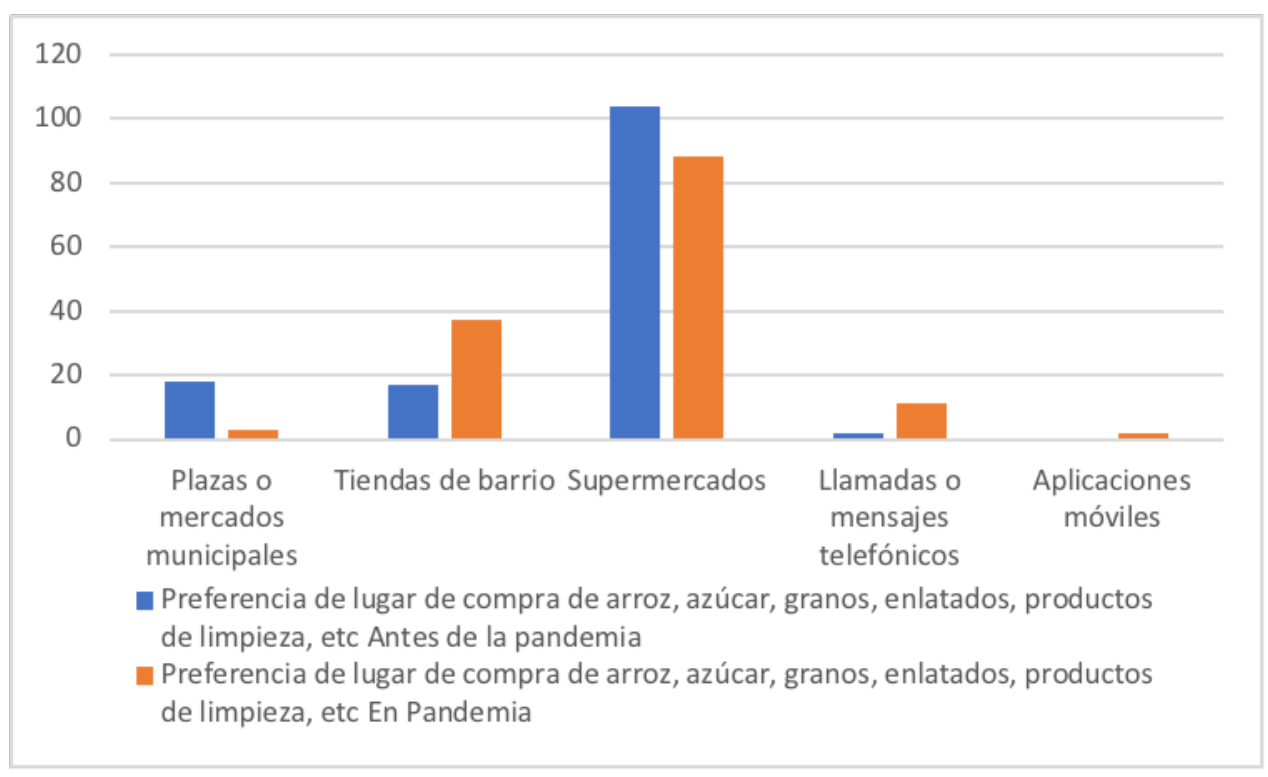

Figura 8. Preferencia de lugar de compra de productos de abasto

Fuente: elaboración propia

De acuerdo la información vertida, es posible afirmar que las tiendas de barrio se han beneficiado de este ajuste de preferencias de lugar de compra en el mercado (Figura 8).

Tabla 3.

Participación etaria

\begin{tabular}{|l|l|l|l|l|l|}
\hline & Frecuencia & Porcentaje & $\begin{array}{l}\text { Porcentaje } \\
\text { válido }\end{array}$ & $\begin{array}{l}\text { Porcentaje } \\
\text { acumulado }\end{array}$ \\
\hline Válido & Entre 22 y 35 & 39 & 28,3 & 28,3 & 28,3 \\
\cline { 2 - 6 } & Entre 36 y 49 & 36 & 26,1 & 26,1 & 54,3 \\
\cline { 2 - 6 } & Entre 50 y63 & 43 & 31,2 & 31,2 & 85,5 \\
\cline { 2 - 6 } & Entre 63 y 77 & 20 & 14,5 & 14,5 & 100 \\
\cline { 2 - 6 } & Total & 138 & 100 & 100 & \\
\hline
\end{tabular}

Fuente: elaboración propia

En correspondencia con la Tabla 3 se evidencia que el grupo de mayor concentración se encuentra entre 50 y 63 años; sin embargo, no existe mucha diferencia con los grupos de menor edad, cuya diferencia está entre 5 y $3 \%$ aproximadamente. Mientras que, el grupo con los de mayor edad son el más pequeño, pero esto no constituye un sesgo para la presente investigación por considerar que, en las condiciones de pandemia, las personas de mayor edad, no son las personas elegidas para salir de compras; sin embargo, estos podrían comprar mediante la aplicación de mecanismos electrónicos, en el caso de que dispongan de recursos tecnológicos, y estén familiarizados con su uso. 
Tabla 4.

Participación por género

\begin{tabular}{|l|l|l|l|l|l|}
\hline \multicolumn{2}{|c|}{} & Frecuencia & Porcentaje & $\begin{array}{l}\text { Porcentaje } \\
\text { válido }\end{array}$ & $\begin{array}{l}\text { Porcentaje } \\
\text { acumulado }\end{array}$ \\
\hline \multirow{4}{*}{ Válido } & Femenino & 71 & 51,4 & 51,4 & 51,4 \\
\cline { 2 - 6 } & Masculino & 67 & 48,6 & 48,6 & 100 \\
\cline { 2 - 6 } & Total & 138 & 100 & 100 & \\
\hline
\end{tabular}

Fuente: elaboración propia

Con estos resultados se corrobora que la muestra tuvo una estructura equitativa en cuanto al género se refiere, tal como se ve en la Tabla 4.

Tabla 5.

Participación por nivel de educación

\begin{tabular}{|l|l|l|l|l|l|}
\hline \multicolumn{2}{|c|}{} & Frecuencia & Porcentaje & $\begin{array}{l}\text { Porcentaje } \\
\text { válido }\end{array}$ & $\begin{array}{l}\text { Porcentaje } \\
\text { acumulado }\end{array}$ \\
\hline \multirow{4}{*}{ Válido } & Básica & 1 & 0,7 & 0,7 & 0,7 \\
\cline { 2 - 6 } & Bachillerato & 14 & 10,1 & 10,1 & 10,9 \\
\cline { 2 - 6 } & Superior & 87 & 63 & 63 & 73,9 \\
\cline { 2 - 6 } & Posgrado & 36 & 26,1 & 26,1 & 100 \\
\cline { 2 - 6 } & Total & 138 & 100 & 100 & \\
\hline
\end{tabular}

Fuente: elaboración propia

En virtud del modo en que se aplicó la presente encuesta, se observa que hubo mayor acceso a personas con un alto nivel de educación (Tabla 5), que sin duda este es un segmento que conoce cómo llenar un formulario en línea, además de que tienen facilidad al acceso tecnológico.

Tabla 6.

¿Está actualmente trabajando?

\begin{tabular}{|l|l|l|l|l|l|}
\hline \multicolumn{2}{|c|}{} & Frecuencia & Porcentaje & $\begin{array}{l}\text { Porcentaje } \\
\text { válido }\end{array}$ & $\begin{array}{l}\text { Porcentaje } \\
\text { acumulado }\end{array}$ \\
\hline \multirow{4}{*}{ Válido } & Sí & 97 & 70,3 & 70,3 & 70,3 \\
\cline { 2 - 6 } & No & 41 & 29,7 & 29,7 & 100 \\
\cline { 2 - 6 } & Total & 138 & 100 & 100 & \\
\hline
\end{tabular}

Fuente: elaboración propia

En la Tabla 6 se evidencia que, si una tercera parte de los encuestados no están trabajando, es posible que ellos no sean actores directos en los procesos de compra; sin embargo, sus respuestas son importantes, en razón de que, tienen amplitud de acción en la influencia de las decisiones de compra en sus familias. 
Tabla 7.

Tipo de empleo

\begin{tabular}{|c|c|c|c|c|c|}
\hline & & Frecuencia & Porcentaje & $\begin{array}{c}\text { Porcentaje } \\
\text { válido }\end{array}$ & $\begin{array}{l}\text { Porcentaje } \\
\text { acumulado }\end{array}$ \\
\hline \multirow[t]{5}{*}{ Válido } & Empleado público & 24 & 17,4 & 19,8 & 19,8 \\
\hline & Empleado privado & 38 & 27,5 & 31,4 & 51,2 \\
\hline & Negocio propio & 36 & 26,1 & 29,8 & 81 \\
\hline & $\begin{array}{l}\text { Nuevo emprendimiento } \\
\text { en este año }\end{array}$ & 23 & 16,7 & 19 & 100 \\
\hline & Total & 121 & 87,7 & 100 & \\
\hline Perdidos & Sistema & 17 & 12,3 & & \\
\hline \multicolumn{2}{|r|}{ Total } & 138 & 100 & & \\
\hline
\end{tabular}

Fuente: elaboración propia

Este instrumento permite recabar información referente al tipo de empleo del encuestado (Tabla 7) lo que permite llevar a cabo investigaciones en donde este aspecto constituya una variable primordial dentro de la investigación.

Tabla 8.

Comportamiento de los ingresos a partir de la pandemia

\begin{tabular}{|c|c|c|c|c|c|}
\hline \multicolumn{2}{|c|}{} & Frecuencia & Porcentaje & $\begin{array}{c}\text { Porcentaje } \\
\text { válido }\end{array}$ & $\begin{array}{c}\text { Porcentaje } \\
\text { acumulado }\end{array}$ \\
\hline \multirow{3}{*}{ Válido } & Se mantuvieron & 34 & 24,6 & 24,6 & 24,6 \\
\cline { 2 - 6 } & Aumentaron & 6 & 4,3 & 4,3 & 29 \\
\cline { 2 - 6 } & Disminuyeron & 98 & 71 & 71 & 100 \\
\cline { 2 - 6 } & & & & & \\
\cline { 2 - 6 } & Total & 138 & 100 & 100 & \\
\hline
\end{tabular}

Fuente: elaboración propia

De acuerdo a los datos recolectados referentes al incremento o disminución de ingresos durante la pandemia dan la oportunidad de determinar movimientos de la economía, nuevas orientaciones al gasto y nuevos emprendimientos y modos de supervivencia (Tabla 8). Esta información es muy valiosa, dado que daría pie a la incursión de nuevas investigaciones.

\section{Conclusiones}


El instrumento diseñado respondió positivamente, tanto a la validación de expertos, como a la validación estadística, dando lugar a la obtención de resultados razonables y con ello se ofrece la oportunidad de su aplicación en diversas investigaciones orientadas a las temáticas en torno a la pandemia 2020. Así en función de la aplicación de este instrumento en la ciudad de Ambato, se recopilaron datos que permiten establecer algunas conclusiones.

En todos los casos, la participación de las ofertas por medio de aplicaciones móviles es secundaria. Aunque pueda considerarse que fue un fenómeno de auge durante los momentos más estrictos de la cuarentena, y que perderá protagonismo conforme las medidas de confinamiento disminuyan, debe tomarse en cuenta que esta constituyó una penetración de mercado acelerada cuyos efectos influirán en la velocidad de adopción de nuevas tecnologías. A pesar de las grandes catástrofes macroeconómicas en curso, y las desgarradoras tragedias en las finanzas domésticas de muchas familias, el $24.1 \%$ de la muestra estudiada ha conseguido mantener sus ingresos, y el $4.1 \%$ incluso los ha aumentado.

Se recomienda poner especial atención en el análisis del comportamiento del segmento cuyos ingresos no se han visto disminuidos durante la pandemia, pues es de considerar que aunque este segmento no se ha visto afectado en su liquidez, si se comprueba por medio de un análisis de variables cruzadas una modificación en su comportamiento, esta posiblemente encontrará su explicación en algunas de las otras condiciones que se han visto afectadas por este particular fenómeno global, como las expectativas del futuro, el temor a la crisis económica o al contagio de COVID-19.

La herramienta puede ser modificado y mejorado en función de las necesidades de los investigadores y abre la oportunidad de iniciar nuevas investigaciones a merced de los intereses de la comunidad académica y científica. 


\section{Referencias}

Bigné, E., Andreu, L., Chumpitaz, R., \& Swaen, V. (2006). La influencia de la responsabilidad social corporativa en el comportamiento de compra de estudiantes universitarios. Esic Market, 6(597), 163-189.

Clavellina Miller, J. L., \& Domínguez Rivas, M. I. (2020). Implicaciones económicas de la pandemia por COVID-19 y opciones de política.

Fernández, E., \& Moreno, J. B. (2001). Pesimismo defensivo, optimismo y dificultad de la tarea: El papel de las expectativas. Revista de psicología general y aplicada: Revista de la Federación Española de Asociaciones de Psicología, 54(3), 371-388.

Gourinchas, P. O. (2020). Flattening the pandemic and recession curves. Mitigating the COVID Economic Crisis: Act Fast and Do Whatever, 31.

Hatzius, J., Philips, A., Mericle, D., \& Struyven, D. (2020). US Daily: a sudden stop for the US economy. Economic Research in Goldman Sachs.

INEC. (2010). Censo de Población y Vivienda. Quito: Instituto Nacional de Estadísticas y Censos.

Levin, R. (2004). Estadística para Administración Economía. México: Pearson Educación: McGraw-Hill.

Lind, D, Marchar W \&Wathen, S. (2005). Estadística Aplicada a los Negocios y

Economía. México: Pearson Educación: McGraw-Hill.

Maldonado, C. E. (2016). El evento raro: Epistemología y complejidad. Cinta de moebio, (56), 187-196. https:// doi.org/10.4067/S0717-554X2016000200006

Martínez, A. I. (2002). Temperamento, carácter; personalidad. Una aproxímacíon a su concepto e interacción. Revista complutense de educación, 13(2), 617.

Nicola, M., Alsafi, Z., Sohrabi, C., Kerwan, A., Al-Jabir, A., losifidis, C., ... \& Agha, R. (2020). The socio-economic implications of the coronavirus pandemic (COVID-19): A review. International journal of surgery (London, England), 78, 185. https://doi.org/10.1016/j.ijsu.2020.04.018

Rodríguez, E. M. R. (2015). Comprensión teórica y proceso metodológico de la investigación cualitativa. In Crescendo, 6(2), 169-183.

Weber, M. (2012). Sociología de la religión (Vol. 222). Ediciones AKAL. 